\title{
Occupational exposure as risk factor for kidney and bladder cancer: a systematic review and meta- analysis
}

\begin{abstract}
Background: Occupational exposure plays a huge role in the epidemiology, pathogenesis and prevalence of kidney cancer $(\mathrm{KCa})$ and bladder cancer $(\mathrm{BCa})$ worldwide.

Objective: The aim of this study was to analyze qualitatively and quantitatively the association between occupational exposure and risk of $\mathrm{KCa}$ and $\mathrm{BCa}$.

Method: We identified peer-reviewed articles published in English by searching PubMed, Embase, Surveillance, Epidemiology, and End Results (SEER) and Cochrane databases. We selected articles published between January 2018 to May 2019. We summed up all relative risk estimates to ensure accuracy, comprehensiveness and maximize statistical power given the low absolute occupational risk of $\mathrm{KCa}$, occupational risk of $\mathrm{BCa}$ and standardized incidence ratios (SIRs). We reported this systematic review and meta-analysis in accordance with the Preferred Reporting Items for Systematic Reviews and metaanalyses (PRISMA) checklist. Quantitative analyzes was performed using Comprehensive Meta-Analysis version 3 (Biostat, Inc, Englewood, New Jersey, USA).

Results:We found no significant association between occupational exposure and $\mathrm{KCa}(\mathrm{OR}$ 1.04, 95\% CI: 0.94-1.34), but there was a significant association between occupational exposure and $\mathrm{BCa}(\mathrm{OR} 1.54,95 \% \mathrm{CI}: 1.44-1.75)$.

Conclusion:From the result of our qualitative and quantitative analysis there was no significant association between occupational exposure and $\mathrm{KCa}$ whereas there was a significant association between occupational exposure and $\mathrm{BCa}$.
\end{abstract}

Keywords: carcinoma, renal cell, kidney neoplasms, urinary bladder neoplasms, occupational exposure, neoplasms
Volume 7 Issue 6 - 2019

\author{
Abdalla Ali Deb,' Chidiebere Emmanuel \\ Okechukwu, ${ }^{2}$ Shady Emara, ${ }^{3}$ Sami A Abbas ${ }^{4}$ \\ 'Locum Consultant Urologist in NHS - UK \\ 2Physical activity and health promotion, Department of \\ Biomedicine and prevention, Faculty of Medicine and Surgery, \\ University of Rome Tor vergata, Italy \\ ${ }^{3}$ Urology Fellow, Western General Hospital, UK \\ ${ }^{4}$ Consultant Urologist; National Institute of Urology and \\ Nephrology, Egypt
}

Correspondence: Abdalla Ali Deb, Locum Consultant Urologist in NHS - UK, Email drabdodeeb@hotmail.com

Received: November 29, 2019 | Published: December 09, 2019
Abbreviations: $\mathrm{KCa}$, kidney cancer; $\mathrm{BCa}$, bladder cancer; SEER, surveillance, epidemiology, and end results; SIRs, standardized incidence ratios; PRISMA, preferred reporting items for systematic reviews and meta-analyses; RCC, renal cell carcinoma; $\mathrm{CI}$, confidence intervals; SIRs, standardized incidence ratios; OR, odd's ratio

\section{Introduction}

Occupational exposure continues to play a huge role in the epidemiology and pathogenesis of cancer worldwide, the prevalence of kidney cancer $(\mathrm{KCa})$ and bladder cancer $(\mathrm{BCa})$ in France is heavily attributed to occupational hazards and exposure to industrial chemicals. ${ }^{1}$ Exposure to industrial chemicals like amines, chromates, dinitrotoluene, arsenic, beryllium, cadmium, nickel, wood dust, crystalline silica, brown coal phosphors, furnace emissions, smoke from diesel engine, ionizing radiation and non-ionizing radiation, thermal shock Asbestos and aniline are major occupational risk factors of $\mathrm{KCa}$ and $\mathrm{BCa}$, also regular shift work, stress and sedentary job contributes to the development of $\mathrm{KCa}$ and $\mathrm{BCa} .{ }^{2}$ Exposure to perchloroethylene mainly used for dry cleaning of fabrics by dry cleaners was associated with $\mathrm{KCa}^{3}{ }^{3}$ The risk of renal cell carcinoma (RCC) was significant in aircraft mechanics, shipbuilders and painters, who were exposed to industrial chemicals. ${ }^{4}$ Exposure to pesticide among farmers may have significant association with kidney cancer. ${ }^{5}$ Traces of heavy metals such as Nickel (Ni), Chromium (Cr) ,Iron (Fe), tungsten (W), titanium (Ti), Cadmium (Cd), Copper (Cu), Manganese (Mn), Zinc ( $\mathrm{Zn})$ particles were found in the analysis of tumor tissue samples using spectrum of X-ray microanalysis. ${ }^{6}$ This shows that heavy metals plays a vital role in the pathogenesis of cancer, Welders are frequently exposed to these heavy metals, and this suggests that they are highly predisposed to $\mathrm{KCa}$ and $\mathrm{BCa}^{6}$

$\mathrm{KCa}$ was ranked the ninth and fourteenth common cancer cases in men and women, respectively. ${ }^{7}$ Apart from occupational exposures, environmental exposures and behavioral factors are associated with the development of $\mathrm{KCa} .{ }^{8} \mathrm{Highly}$ skilled workers in some occupation that involves little or no exposures to stress and chemicals often have less chances of developing RCC. ${ }^{9}$ Consumption of fruit, vegetables, and alcohol was linked with a decreased risk of RCC despite the presence or exposure to the risk factors..$^{10}$ High levels of arsenic in the urine was associated with the pathogenesis $\mathrm{KCa} .{ }^{11}$ Exhaust from diesel engine is associated with the pathogenesis of RCC. ${ }^{12} \mathrm{KCa}$ is prevalent in the United States due to occupational exposures. ${ }^{13} \mathrm{BCa}$ is prevalent worldwide, Exposure to heavy industrial chemicals like trichloroethylene, perchloroethylene, aromatic hydrocarbon solvents, benzene and toluene in the working environment and cigarette 
smoking are the main risk factors of $\mathrm{BCa} .{ }^{14} \mathrm{Kishor}$ et al. ${ }^{8,15}$ observed statistically significant increased risks of $\mathrm{BCa}$ among tobacco workers, plumbers, chimney sweeps, waiters, hairdressers, seamen, printers and they observed Significant decrease in risks among farmers and forestry workers. There is need to critically analyze and review the occupational risk factors associated with $\mathrm{KCa}$ and $\mathrm{BCa}$ for the purpose of primary prevention and maintaining occupational safety and health. The aim of this study was to analyze systematically and quantitatively the association between various occupational exposures and risk of $\mathrm{KCa}$ and $\mathrm{BCa}$.

\section{Methods}

We performed and reported this systematic review and metaanalysis in accordance with the Preferred Reporting Items for Systematic Reviews and meta-analyses (PRISMA) checklist. ${ }^{16}$

\section{Search strategy}

We identified peer-reviewed articles published in English by searching PubMed, Embase, Surveillance, Epidemiology, and End Results (SEER) and Cochrane databases. We selected articles published between January 2018 to May 2019. The following search terms was generally used; $\mathrm{KCa}$, renal cancer and occupational exposure, $\mathrm{BCa}$ and occupational exposure, neoplasms, and tumor. First, we screened all titles and abstracts we read the full text of relevant studies. We selected all available studies related to occupational exposure in relation to $\mathrm{KCa}$ and $\mathrm{BCa}$. With regards to $\mathrm{MeSH}$ terms and relevant keywords (Carcinoma, Renal Cell, Kidney Neoplasms, Urinary Bladder Neoplasms, Occupational Exposure, Neoplasms), we used the Cochrane Highly Sensitive Search Strategy for identifying reports of articles in PubMed. We restricted the search to articles published in English.

\section{Study eligibility}

Studies were included if: 1 . they evaluate occupation exposures as risk factors for $\mathrm{KCa}$ and $\mathrm{BCa} 2$. They were designed as cohort, case-control, cohort-case control, cross-sectional, or ecological studies 3.They provided data on $\mathrm{KCa}$ and $\mathrm{BCa}$ risks associated with occupation exposures. Studies were excluded if: 1. they were not conducted on humans 2 . They were designed as individual case reports 3 . They did not contain original data and instead provided reviews, reanalysis, or commentaries and 4 . They did not analyze the role of occupational exposures in the pathogenesis of $\mathrm{KCa}$ and $\mathrm{BCa}$.

\section{Data extraction}

We extracted the following data of each eligible study onto a spreadsheet: first author, publication year, location, study type, follow-up period, study population, sample size, type of occupational exposure, numbers of $\mathrm{KCa}$ cases reported, number of $\mathrm{BCa}$ cases reported, association measures and their $95 \%$ confidence intervals (CI), and controlled confounding factors.

\section{Statistical analysis}

We summed up all relative risk estimates to ensure accuracy, comprehensiveness and maximize statistical power given the low absolute occupational risk of $\mathrm{KCa}$; occupational risk of $\mathrm{BCa}$, standardized incidence ratios (SIRs). We applied Q test and $\mathrm{I}^{2}$ index to evaluate heterogeneity across studies. Q test $\mathrm{P}$ values of less than 0.1 indicate significant heterogeneity, and $\mathrm{I}^{2}$ values of near or less than $25 \%$, near $50 \%$, and nearor higher than $75 \%$ represent low, moderate, andhigh heterogeneity, respectively. We performed analyzes based on study designs and populations to investigate possible sources of heterogeneity.We made use of the following tools: 1. Pooled OR: for analysis of multiple risks and Odd's ratio (OR) of multiple studies, and found adjusted accumulative OR for all studies 2. Fisher's method: Combining $\mathrm{p}$ values from independent tests bearing upon the same overall hypotheses $3 . \mathrm{Z}$ score method: to test difference in mean 4. Test for heterogeneity: Cochran's Q test and I'2: Under null, it is approximately, distributed as a chi-square with k-1 degrees of freedomfor test heterogeneity and homogeneity of studies results and finding. All analyzes were performed using Comprehensive MetaAnalysis version 3.3.070 (Biostat, Inc, Englewood, New Jersey, USA).

\section{Results}

\section{Study selection}

The search yielded 15,110 articles in total. After removing duplicates, 7,569 articles remained. We excluded 2,340 articles because their abstracts were irrelevant with regards to the topic of our study. The references of the 5229 remaining articles were searched for full-text reviews. We excluded 1254 studies with unclear results, 1986 reanalysis studies, 1392 studies that did not report the outcome of occupational exposure as risk factors for and $\mathrm{BCa}, 461$ studies with inadequate evidence and 120 study with unavailable full text. The remaining 19 studies were systematically reviewed, while 16 studies were included for meta-analysis (Figure 1).

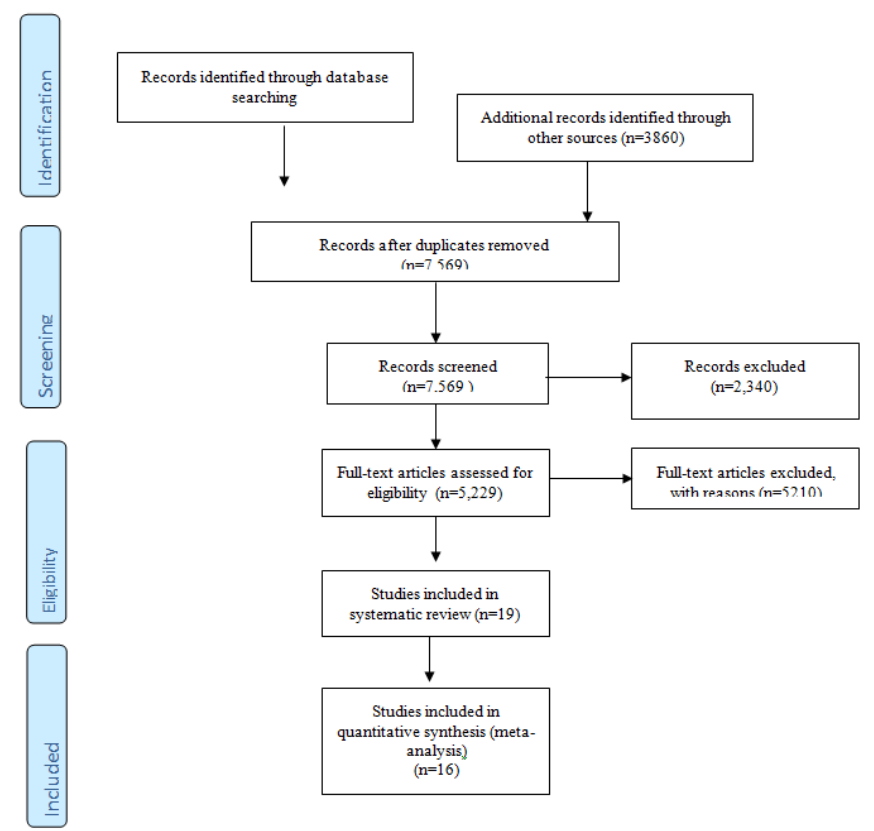

Figure I Preferred Reporting Items for Systematic Reviews and metaanalyses (PRISMA).

\section{Study characteristics}

As shown in Table 1 and Table 2, the studies that was selected for the systematic review and meta-analysis were published between January 2018 and May 2019. We qualitatively analyzed these articles in order to ascertain the association between occupational exposure and risk of $\mathrm{KCa}$ and $\mathrm{BCa}$. 
Table I Occupational exposure and risk of kidney cancer

\begin{tabular}{|c|c|c|c|c|c|c|c|c|}
\hline Study & Country & Study type & Follow-up & $\begin{array}{l}\text { Study } \\
\text { population }\end{array}$ & Sample size & Case & Increased risks $(95 \% \mathrm{CI})$ & Outcome \\
\hline $\begin{array}{l}\text { Michelek } \\
\text { et al. }{ }^{17}\end{array}$ & $\begin{array}{l}\text { Finland, } \\
\text { Iceland, } \\
\text { and } \\
\text { Sweden }\end{array}$ & $\begin{array}{l}\text { Case-control } \\
\text { study }\end{array}$ & $1960-1990$ & $\begin{array}{l}\text { population } \\
\text { censuses }\end{array}$ & $\begin{array}{l}59,778 \mathrm{KCa} \text { cases, and } \\
298,890 \text { controls }\end{array}$ & 59,778 & $\begin{array}{l}\text { Individuals with high exposure } \\
\text { to asbestos (OR I. } 19,95 \% \mathrm{Cl} \\
\text { I.08-I.3I). } \\
\text { Individuals characterized } \\
\text { with high perceived physical } \\
\text { workload (OR } 0.86,95 \% \mathrm{Cl} \\
0.82-0.9 \mathrm{I}) \text {. } \\
\text { Individual with high exposure } \\
\text { to ultraviolet radiation (OR } \\
0.85,95 \% \mathrm{Cl} 0.79-0.92) \text {. } \\
\text { Individuals with high exposure } \\
\text { to wood dust (OR } 0.82,95 \% \mathrm{Cl} \\
0.7 \mathrm{I}-0.94) \text {. }\end{array}$ & $\begin{array}{l}\text { High risk of } \mathrm{KCa} \\
\text { was observed } \\
\text { among individuals } \\
\text { exposed to } \\
\text { welding fumes. }\end{array}$ \\
\hline $\begin{array}{l}\text { Michelek } \\
\text { et al. }{ }^{18}\end{array}$ & $\begin{array}{l}\text { Nordic } \\
\text { population }\end{array}$ & $\begin{array}{l}\text { Case-cohort } \\
\text { study }\end{array}$ & $1960-1990$ & $\begin{array}{l}\text { population } \\
\text { censuses }\end{array}$ & 14.9 million & 59,778 & $\begin{array}{l}\text { SIRs: welders }[1.24,(95 \% \mathrm{Cl}) \\
\text { I.I } 4 \text { to I.35], public safety } \\
\text { workers }(1.16,95 \% \mathrm{Cl} \mathrm{I.08} \mathrm{to} \\
\text { I.25). Seamen }(1.16,95 \% \mathrm{Cl} \\
\text { I.07 to I.26). } \\
\text { Significantly decreased SIRs } \\
\text { were found in lab assistants } \\
(0.76,95 \% \mathrm{Cl} 0.60 \text { to } 0.94) \text { and } \\
\text { forestry workers }(0.77,95 \% \mathrm{Cl} \\
0.72 \text { to } 0.83) \text {. }\end{array}$ & $\begin{array}{l}\text { Variation in the } \\
\text { incidence of } \mathrm{KCa} \\
\text { was observed. }\end{array}$ \\
\hline $\begin{array}{l}\text { Zaitsuet } \\
\text { al. }{ }^{19}\end{array}$ & Japan & $\begin{array}{l}\text { Case-cohort } \\
\text { study }\end{array}$ & $\begin{array}{l}1984 \text { to } \\
2016\end{array}$ & $\begin{array}{l}\text { Nationwide } \\
\text { inpatient records }\end{array}$ & $\begin{array}{l}3316 \text { cases of renal cell } \\
\text { cancer (excluding upper } \\
\text { tract urothelial cancer) } \\
\text { and } 168418 \text { controls. }\end{array}$ & 3316 & (OR, I.6I; 95\% Cl, I.34-1.93). & $\begin{array}{l}\text { Occupational class } \\
\text { is associated with } \\
\text { the risk of } \mathrm{KCa} \\
\text { in men. }\end{array}$ \\
\hline $\begin{array}{l}\text { Peters } \\
\text { et al. }{ }^{20}\end{array}$ & Canada & $\begin{array}{l}\text { Case-control } \\
\text { study }\end{array}$ & $\begin{array}{l}1994 \text { and } \\
1997\end{array}$ & $\begin{array}{l}\text { National } \\
\text { Enhanced Cancer } \\
\text { Surveillance } \\
\text { System }\end{array}$ & $\begin{array}{l}712 \text { cases and } 2454 \\
\text { controls }\end{array}$ & 712 & (OR I.2, 95\% Cl, I.0-1.4). & $\begin{array}{l}\text { High level of } \\
\text { exposure to } \\
\text { asbestos is } \\
\text { associated with } \\
\mathrm{KCa} \text { risk }\end{array}$ \\
\hline $\begin{array}{l}\text { Peters } \\
\text { et al. }{ }^{21}\end{array}$ & Canada & $\begin{array}{l}\text { Case- } \\
\text { control } \\
\text { study }\end{array}$ & $1994-1997$ & $\begin{array}{l}\text { National } \\
\text { Enhanced Cancer } \\
\text { Surveillance } \\
\text { System (NECSS) }\end{array}$ & $\begin{array}{l}712 \text { cases and } 2457 \\
\text { cases }\end{array}$ & 712 & $\begin{array}{l}\text { (OR diesel=I.23, } 95 \% \\
\mathrm{Cl}=0.99-\mathrm{I} .53 ; \mathrm{OR} \\
\text { gasoline }=\mathrm{I} .5 \mathrm{I}, 95 \% \mathrm{Cl}=\mathrm{I} .23- \\
\mathrm{I} .86 \text { ). }\end{array}$ & $\begin{array}{l}\text { Occupational } \\
\text { gasoline and diesel } \\
\text { exhaust exposure } \\
\text { may increase the } \\
\text { risk of KCa. }\end{array}$ \\
\hline $\begin{array}{l}\text { Saint- } \\
\text { Jacques } \\
\text { et al. }{ }^{22}\end{array}$ & Canada & $\begin{array}{l}\text { Ecological } \\
\text { study }\end{array}$ & $1998-2010$ & $\begin{array}{l}\text { inpatient } \\
\text { records- Nova } \\
\text { Scotia, Canada }\end{array}$ & $\begin{array}{l}864 \text { bladder and } 525 \\
\text { kidney cases }\end{array}$ & $\begin{array}{l}864 \\
525\end{array}$ & - & $\begin{array}{l}\text { This study suggests } \\
\text { an increased KCa } \\
\text { associated risk } \\
\text { from drinking } \\
\text { water with } \\
\text { increased arsenic- } \\
\text { levels }\end{array}$ \\
\hline $\begin{array}{l}\text { Michalek } \\
\text { et al. }{ }^{23}\end{array}$ & $\begin{array}{l}\text { Denmark } \\
\text { Iceland } \\
\text { Finland } \\
\text { Norway } \\
\text { Sweden }\end{array}$ & $\begin{array}{l}\text { Cohort } \\
\text { study }\end{array}$ & - & $\begin{array}{l}\text { National } \\
\text { censuses }\end{array}$ & 14.9 million & 59,778 & 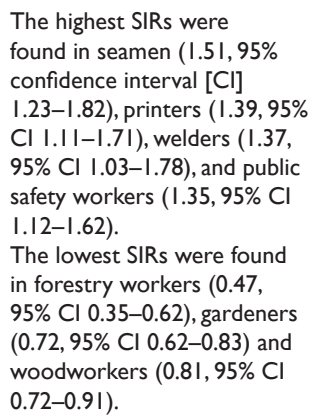 & $\begin{array}{l}\text { There was an } \\
\text { association } \\
\text { between } \\
\text { profession and risk } \\
\text { of malignancy of } \\
\text { the renal pelvis. }\end{array}$ \\
\hline $\begin{array}{l}\text { Callahan } \\
\text { et al. } .^{24}\end{array}$ & USA & $\begin{array}{l}\text { Case-control } \\
\text { study }\end{array}$ & $\begin{array}{l}2002 \text { and } \\
2007\end{array}$ & $\begin{array}{l}\text { Lead industry } \\
\text { workers }\end{array}$ & $\begin{array}{l}1217 \text { cases and } 1235 \\
\text { controls cases and } 1235 \\
\text { controls }\end{array}$ & 1217 & $\begin{array}{l}\text { (OR } 0.9,95 \% \mathrm{Cl} 0.7 \text { to } 1.3 \text { for } \\
\text { highest quartile vs unexposed; } \\
\text { ptrend }=0.80 \text { ). }\end{array}$ & $\begin{array}{l}\text { No association was } \\
\text { found between } \\
\text { occupational lead } \\
\text { exposure and } \mathrm{KCa}\end{array}$ \\
\hline
\end{tabular}

Cis, confidence intervalsORs, odds ratios SIRs,standardized incidence ratios

Citation: Deb AA, Okechukwu CE, Emara S, et al. Occupational exposure as risk factor for kidney and bladder cancer: a systematic review and meta-analysis. Urol Nephrol Open Access J. 20 I9;7(6): I43-15I. DOI: 10.15406/unoaj.2019.07.0026 I 
Table 2 Occupational exposures and risk of bladder cancer

\begin{tabular}{|c|c|c|c|c|c|c|c|c|}
\hline Study & Country & Study type & Follow-up & $\begin{array}{l}\text { Study } \\
\text { population }\end{array}$ & Sample size & Case & Increased risks $(95 \% \mathrm{Cl})$ & Outcome \\
\hline $\begin{array}{l}\text { Carey et } \\
\text { al. }^{25}\end{array}$ & $\begin{array}{l}\text { Denmark } \\
\text { Finland } \\
\text { Iceland } \\
\text { Norway } \\
\text { Sweden }\end{array}$ & $\begin{array}{l}\text { Cohort } \\
\text { study }\end{array}$ & $196 \mid-2005$ & $\begin{array}{l}\text { Nordic } \\
\text { Occupational } \\
\text { Cancer study } \\
\text { (NOCCA) }\end{array}$ & 111,458 & 111,458 & 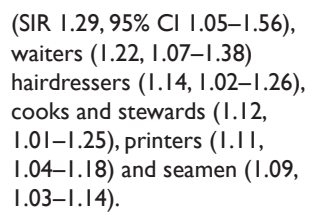 & $\begin{array}{l}\text { Smoking is a strong } \\
\text { risk factor for } \mathrm{BCa} \text {. }\end{array}$ \\
\hline $\begin{array}{l}\text { Turner et } \\
\text { al. } .^{26}\end{array}$ & Spain & $\begin{array}{l}\text { Case-control } \\
\text { study }\end{array}$ & $|998-200|$ & $\begin{array}{l}\text { Large-scale } \\
\text { Spanish BCa } \\
\text { Study. }\end{array}$ & $\begin{array}{l}938 \text { cases and } 973 \\
\text { controls }\end{array}$ & 938 & $\begin{array}{l}\text { (OR per } 5.9 \mu \mathrm{g} / \mathrm{m} 3=1.06,95 \% \\
\mathrm{Cl} 0.71-1.60 \text { ) or } \mathrm{NO} 2(\mathrm{OR} \\
\text { per } 14.2 \mu \mathrm{g} / \mathrm{m} 3=0.97,95 \% \mathrm{Cl} \\
0.84-1.13 \text { ) }\end{array}$ & $\begin{array}{l}\text { There was no } \\
\text { clear evidence for } \\
\text { associations of } \\
\text { ambient particulate } \\
\text { matter (PM2.5) and } \\
\text { nitrogen dioxide } \\
\text { (NO2) concentrations } \\
\text { and incident BCa risk. }\end{array}$ \\
\hline $\begin{array}{l}\text { Makiko et } \\
\text { al. }{ }^{27}\end{array}$ & Japan & $\begin{array}{l}\text { cross- } \\
\text { sectional } \\
\text { study }\end{array}$ & 2017 & $\begin{array}{l}76 \text { ortho- } \\
\text { toluidine (OT) } \\
\text { and/or aromatic } \\
\text { amine-exposed } \\
\text { workers }\end{array}$ & 76 & 10 & - & $\begin{array}{l}\text { OT-exposed workers } \\
\text { had histories of gross } \\
\text { hematuria and cystitis. }\end{array}$ \\
\hline $\begin{array}{l}\text { Marant et } \\
\text { al. }{ }^{28}\end{array}$ & France & $\begin{array}{l}\text { Cohort } \\
\text { study }\end{array}$ & 2015 & $\begin{array}{l}\text { Surveys among } \\
\text { employees, } \\
\text { the national } \\
\text { labor force } \\
\text { data, a cohort } \\
\text { of agricultural } \\
\text { workers, national } \\
\text { monitoring of } \\
\text { workers }\end{array}$ & 7905 & $\begin{array}{l}7336 \\
\text { among } \\
\text { men } \\
\text { and } 569 \\
\text { among } \\
\text { women }\end{array}$ & - & $\begin{array}{l}\text { BCa was ranked as } \\
\text { the } 3 \text { rd occupation } \\
\text { associated cancer } \\
\text { in men. }\end{array}$ \\
\hline $\begin{array}{l}\text { Carta et } \\
\text { al. }^{29}\end{array}$ & Italy & $\begin{array}{l}\text { cross- } \\
\text { sectional } \\
\text { study }\end{array}$ & - & $\begin{array}{l}\text { Non-Muscle- } \\
\text { Invasive Bladder } \\
\text { Cancer (NMIBC) } \\
\text { patients }\end{array}$ & 160 & 160 & $\begin{array}{l}(H R=0.195 ; 95 \% C l=0.060 \text { to } \\
0.623 ; p=0.006)\end{array}$ & $\begin{array}{l}\text { A mechanism exist } \\
\text { between cause (ValVal } \\
\text { genotype of both } \\
\text { MnSOD and COMT) } \\
\text { and effect (decreased } \\
\text { progression of tumor } \\
\text { in NMIBC patients). }\end{array}$ \\
\hline $\begin{array}{l}\text { Régis et } \\
\text { al. }^{30}\end{array}$ & France & $\begin{array}{l}\text { A nested } \\
\text { case-control } \\
\text { study }\end{array}$ & $\begin{array}{l}2006 \text { to } \\
2012\end{array}$ & $\begin{array}{l}\text { Cohort of } \\
\text { workers from } \\
\text { six French } \\
\text { steel-producing } \\
\text { factories }\end{array}$ & $\begin{array}{l}\text { Cases }(n=84) \text { and } \\
\text { controls }(n=25 I)\end{array}$ & 84 & $\begin{array}{l}(O R=1.13(1.02-1.25) \\
(O R=1.44(0.97-2.14)\end{array}$ & $\begin{array}{l}\text { There is an increased } \\
\text { risk of BCa observed } \\
\text { among workers } \\
\text { exposed to straight } \\
\text { metalworking fluids } \\
\text { (MWFs) }\end{array}$ \\
\hline $\begin{array}{l}\text { Sciannameo } \\
\text { et al. } .^{31}\end{array}$ & Italy & $\begin{array}{l}\text { case-control } \\
\text { study }\end{array}$ & $1992-2012$ & $\begin{array}{l}\text { chemical } \\
\text { engineering } \\
\text { technicians } \\
\text { postmen lathe } \\
\text { operators }\end{array}$ & $\begin{array}{l}893 \text { cases and } 978 \\
\text { control. }\end{array}$ & 893 & - & $\begin{array}{l}\text { A significantly } \\
\text { increased } \mathrm{BCa} \\
\text { risk was found for } \\
\text { chemical engineering } \\
\text { technicians, postmen, } \\
\text { and lathe operators. }\end{array}$ \\
\hline Jung et al..$^{32}$ & Canada & $\begin{array}{l}\text { societal } \\
\text { perspective }\end{array}$ & 2011 & $\begin{array}{l}\text { Diagnosed } \\
\text { cases of } \mathrm{BCa} \text { in } \\
\text { Canada that is } \\
\text { associated with } \\
\text { occupational } \\
\text { exposure }\end{array}$ & 199 & 199 & - & $\begin{array}{l}\text { The economic } \\
\text { burden of } \mathrm{BCa} \text { due } \\
\text { to occupational } \\
\text { exposures is very } \\
\text { enormous }\end{array}$ \\
\hline
\end{tabular}

Citation: Deb AA, Okechukwu CE, Emara S, et al. Occupational exposure as risk factor for kidney and bladder cancer: a systematic review and meta-analysis. Urol Nephrol Open Access J. 20 I9;7(6): I43-I5I. DOI: 10.15406/unoaj.20 I9.07.0026 I 


\begin{tabular}{|c|c|c|c|c|c|c|c|c|}
\hline Study & Country & Study type & Follow-up & $\begin{array}{l}\text { Study } \\
\text { population }\end{array}$ & Sample size & Case & Increased risks $(95 \% \mathrm{Cl})$ & Outcome \\
\hline $\begin{array}{l}\text { Noon et } \\
\text { al. }{ }^{33}\end{array}$ & Finland & $\begin{array}{l}\text { Cohort } \\
\text { study }\end{array}$ & - & $\begin{array}{l}\text { National data } \\
\text { set of workers }\end{array}$ & $\begin{array}{l}\text { I.7 million Finnish } \\
\text { men, I.7 million } \\
\text { women }\end{array}$ & $\begin{array}{l}13717 \\
\text { with } \\
\text { BCa for } \\
\text { men, } \\
4282 \\
\text { with } \\
\text { BCa for } \\
\text { women }\end{array}$ & $\begin{array}{l}\text { Male chemical process } \\
\text { workers (SIRloc/SIRadv: } \\
\text { 5.I9; 95\% CI, I.73-25.7), } \\
\text { male military personnel } \\
\text { (SIRloc/SIRadv: 6.4; 95\% CI, } \\
\text { I.09-259.0), male public } \\
\text { safety workers (SIRloc/SIRadv: } \\
\text { I.77; } 95 \% \text { Cl, I.04-3.23), } \\
\text { Miscellaneous construction } \\
\text { workers (male SIRloc/SIRadv: } \\
\text { 0.67; 95\% Cl, 0.53-0.86; female } \\
\text { SIRloc/SIRadv: 0.12; } 95 \% \mathrm{Cl} \text {, } \\
\text { 0.09-0.54) }\end{array}$ & $\begin{array}{l}\text { Occupations may } \\
\text { differ in their risks } \\
\text { for localized and } \\
\text { advanced BCa. }\end{array}$ \\
\hline $\begin{array}{l}\text { Golka et } \\
\text { al. } .^{34}\end{array}$ & Germany & $\begin{array}{l}\text { case-control } \\
\text { study }\end{array}$ & $\begin{array}{l}\text { July } 2009 \\
\text { to July } \\
2013\end{array}$ & $\begin{array}{l}\text { Former hard } \\
\text { coal miners }\end{array}$ & $\begin{array}{l}400 \text { bladder cancer } \\
\text { cases and } 442 \\
\text { controls }\end{array}$ & 400 & $\begin{array}{l}\text { (OR } 0.96,95 \% \mathrm{Cl}: 0.73 \text { to I.26; } \\
\text { smokers: OR } 0.93,95 \% \mathrm{Cl} \text { : } \\
0.66 \text { to I.30; non-smokers: } \\
\text { OR I.02, } 95 \% \mathrm{Cl}: 0.58 \text { to I.80) } \\
\text { OR } 3.22,95 \% \mathrm{Cl}: 1.39 \text { to } 7.49 \text { ); } \\
\text { hospital B: } 32 \text { cases (I6\%) and } \\
20 \text { controls (I0\%) (OR I.72, } \\
95 \% \mathrm{Cl}: 0.95 \text { to } 3.12 \text { ) }\end{array}$ & $\begin{array}{l}\text { There was an elevated } \\
\text { BCa risk in former } \\
\text { hard coal miners }\end{array}$ \\
\hline $\begin{array}{l}\text { Hameed et } \\
\text { al. }{ }^{35}\end{array}$ & Egypt & $\begin{array}{l}\text { case-control } \\
\text { study }\end{array}$ & - & $\begin{array}{l}\text { Patients } \\
\text { from different } \\
\text { upper Egypt } \\
\text { governorates }\end{array}$ & $\begin{array}{l}100 \text { cases, } 200 \\
\text { controls }\end{array}$ & 100 & - & $\begin{array}{l}\text { The results revealed } \\
\text { that the level of } \\
\text { P53 was significantly } \\
\text { high in comparison } \\
\text { with the control } \\
\text { group }(\mathrm{p}<0.00 \mathrm{I}) \text {. }\end{array}$ \\
\hline
\end{tabular}

Cis, confidence intervals ORs, odds ratios SIRs,standardized incidence ratios

\section{Quality assessment of studies}

For the studies that investigated the association of occupational exposure and risk of $\mathrm{KCa}$; there were 4 case control studies, ${ }^{17,20,21,243}$ cohort studies $18,{ }^{19,23}$ and 1 ecological study ${ }^{22}$ they were all population based studies. For the studies that investigated the association of occupational exposures and risk of bladder cancer; there were 5 case control studies, ${ }^{26,30,31,34,35} 3$ cohort studies, ${ }^{25,28,33} 2$ cross sectional studies, ${ }^{27,29}$ and 1 social perspective. ${ }^{32}$ All studies that reported on occupational exposure as risk factors for $\mathrm{KCa}$ and $\mathrm{BCa}$ Between January 2018 to May 2019 was initially selected for the study. To assess the quality of the studies, STROBE ${ }^{36}$ was used as the standard checklist. This checklist contains 22 items that cover different parts of a report (sampling, measurement of variables, study objectives, and statistical analysis). We gave one point to each item, and some other items that were more important to us had more points. The STROBE checklist contains 22 sections that cover different parts of a report, and the maximum score of a report equals 44 , so that a score of $1-15$ indicates poor quality, 16-30 shows average quality, and 31-44 is considered to be excellent. We excluded 3 articles with an overall score less than 16 for the meta-analysis.

\section{Risk of bias within studies}

Association and risk of occupational exposures for kidney cancer distribution

To assess publication bias a funnel plot was derived, and heterogeneity among studies results was checked. Confounding, interaction, and bias account for differences in results among studies, which are not due to chance, after quantifying, we discovered that it contributed to heterogeneity among these studies. We found significant heterogeneity and disagreement between studies (Table 3).Heterogeneity and asymmetry was illustrated in funnel plot (Figure 2).

Table 3 Significant heterogeneity and disagreement was found between studies

\begin{tabular}{ll}
\hline \multicolumn{2}{l}{ Test for heterogeneity } \\
\hline Cochran Q & 40.32 \\
$P$ & $0.00 * *$ \\
I2 (Inconsistency) & 26.9 \\
$95 \% \mathrm{Cl}$ for 12 & $18.84-91.6$
\end{tabular}

\section{Association and risk of occupational exposure for bladder cancer distribution}

To assess publication bias a funnel plot was derived (Figure 3), homogeneity among studies were founded. No bias accounted for differences in results among studies, which are not due to chance, after quantifying all factors. We found no significant heterogeneity and agreement between studied (Table 4). 


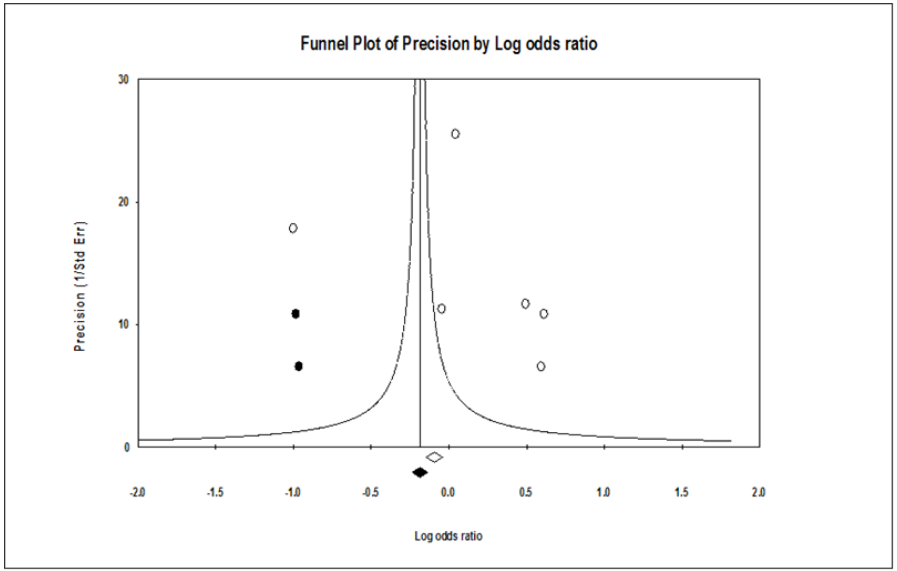

Figure 2Heterogeneity and asymmetry was found and illustrated in funnel plot; Funnel plot of studiesevaluating the association between occupational exposures and $\mathrm{KCa}$. Dotted lines indicate $95 \%$ pseudo-confidence interval. Std Err: standard error and Logs odds ratio.

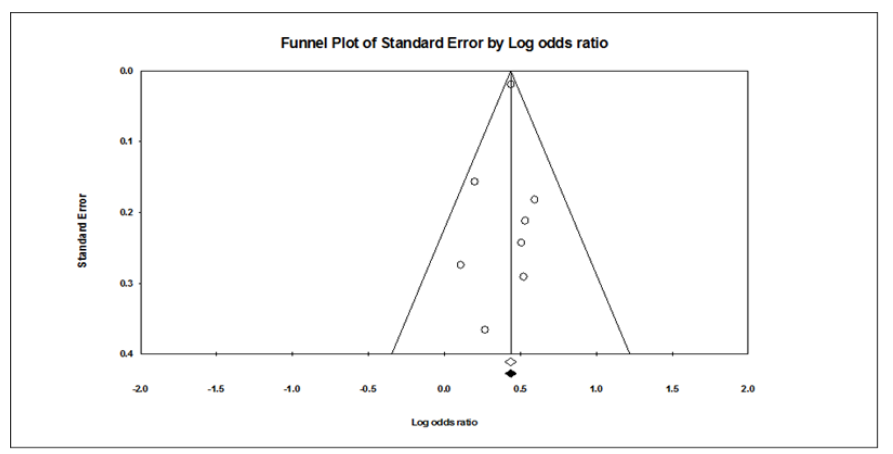

Figure 3Homogeneity and symmetry was found and illustrated in funnel plot; Funnel plot of studies evaluating the association between occupational exposures for $\mathrm{BCa}$. Dotted lines indicate $95 \%$ pseudo-confidence interval. Standard error, Log odds ratio.

Table 4 No significant heterogeneity and agreement was found between studies

\begin{tabular}{ll}
\hline \multicolumn{2}{l}{ Test for heterogeneity } \\
\hline Cochran Q & 5.87 \\
P & 0.215 \\
I2 (Inconsistency) & 6.54 \\
$95 \%$ Cl for I2 & $0.84-8.36$ \\
\hline
\end{tabular}

\section{Categorical meta-analysis findings}

\section{Association and risk of occupational exposure for $\mathrm{KCa}$ distribution}

Table 5shows socio demographic distribution as mean age from all studies was $56.45 \pm 17.6$ and male were majority with average percentage of $95.3 \%$ and about $42.5 \%$ were smokers. Table 6 and Figure 4 shows association and risk of occupational exposure for renal cancer distribution.Peters et al. ${ }^{20}(\mathrm{OR} 1.63,95 \%$ CI: $1.33-1.96)$, Saint-Jacques et al. ${ }^{22}(\mathrm{OR} 1.808,95 \%$ CI: 1.34-2.43) and Michelek et al. ${ }^{23}(\mathrm{OR} 1.842,95 \% \mathrm{CI}: 1.53-2.18)$ showed significant risk but Michelek et al. ${ }^{23}$ (OR 0.81, 95\% CI: 0.71-1.15 ), Zaitsu et al. ${ }^{19}(\mathrm{OR}$
1.04,95\% CI: 0.96-1.12) and Callahan et al. ${ }^{24}$ (0.95, 95\% CI: 0.84 1.37 ) showed no significant risk or association. After analysis of multiple paper regarding the association between $\mathrm{KCa}$ and different occupational exposure we found no significant association (OR 1.04, 95\% CI:0.94-1.34).

Table 5 Distribution of demographic data of kidney cancer studies

\begin{tabular}{|c|c|c|c|c|c|}
\hline Study & $\mathbf{N}$ & Age & Sex & & Smoking \\
\hline & & & Male & Female & \\
\hline Michelek et al. ${ }^{18}$ & 57310 cases & $59.54 \pm 18.65$ & $98.00 \%$ & $2.00 \%$ & $49.60 \%$ \\
\hline Zaitsuet al. ${ }^{19,18}$ & 3316 cases & $57.48 \pm 13.65$ & $81.60 \%$ & $18.40 \%$ & $74.60 \%$ \\
\hline Peters et al. ${ }^{20}$ & 712 cases & $59.0 \pm 16.32$ & $100.00 \%$ & $0.00 \%$ & $16.00 \%$ \\
\hline $\begin{array}{l}\text { Saint-Jacques } \\
\text { et al. }{ }^{20}\end{array}$ & 525 cases & $56.41 \pm 11.8$ & NA & NA & NA \\
\hline Michalek et al. ${ }^{23}$ & 59778 cases & NA & NA & NA & NA \\
\hline Callahan et al. ${ }^{24}$ & 1217 cases & $52.87 \pm 10.85$ & NA & NA & $45.00 \%$ \\
\hline
\end{tabular}

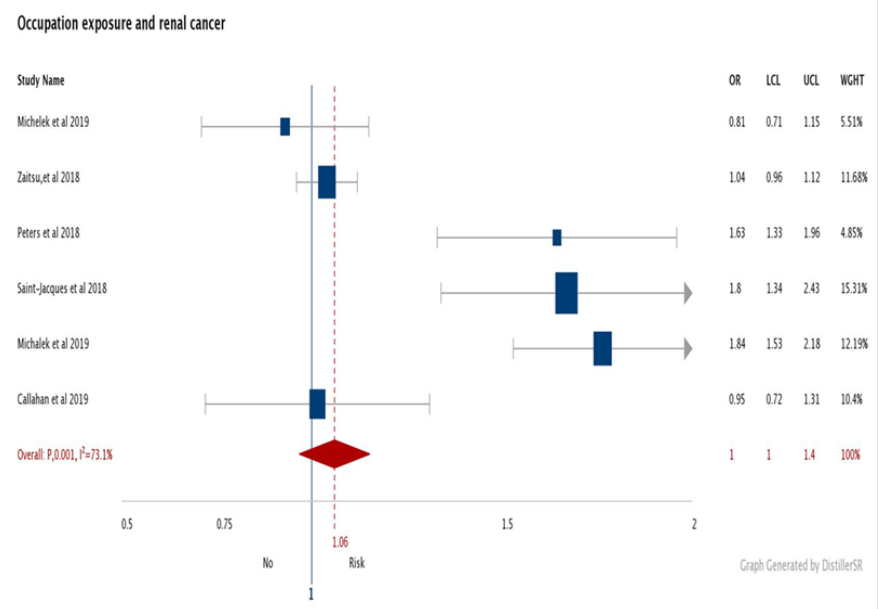

Figure 4 Forest plot of association occupational exposure and $\mathrm{KCa}$. Box sizes reflect the weights of studies included in the meta-analysis, horizontal lines are the $95 \% \mathrm{Cls}$, and the summary OR is represented by the diamond. OR: odds ratio, $\mathrm{Cl}$ : confidence interval.

\section{Association and risk of occupational exposure for $\mathrm{BCa}$ distribution}

Table 6 Socio demographic distribution as mean age from all studies was $59.88 \pm 8.5$ and male were majority with average percentage of $83.3 \%$ and about $48.8 \%$ were smokers. Table 7 and Figure 5 shows association and risk of occupational exposure for $\mathrm{BCa}$ distribution;Carey et al. ${ }^{25}$ (OR 1.55, 95\% CI: 1.38-1.69), Sciannameo et al. ${ }^{31}$ (OR 1.81, 95\% CI: 1.23-2.58), Noon et al..$^{33}$ (OR 1.66, 95\% CI: 1.03-2.67), Golka et al. ${ }^{34}(\mathrm{OR} 1.74,95 \%$ CI: 1.12-2.53) and Hameed et al. ${ }^{35}$ (OR 1.98, 95\% CI: 1.15-2.98) found and illustrated significant risk and association between occupational exposure and $\mathrm{BCa}$ but Turner et al. ${ }^{26}$ (OR 1.22, 95\% CI: 0.86-1.65), Marant et al. ${ }^{28}$ (OR $1.30,95 \%$ CI:0.63-2.69) and Régis et al. ${ }^{30}(\mathrm{OR} 1.11,95 \%$ CI: 0.65 1.98) found no significant risk. After the analysis of multiple paper regarding the association between $\mathrm{BCa}$ and different occupational exposure we found a significant association (OR 1.54,95\% CI:1.441.75) (Table 8). 
Table 6 Association and risk of occupational exposure for KCa distribution

\begin{tabular}{|c|c|c|c|c|c|}
\hline & & & $\mathbf{z}$ & $\mathbf{P}$ & \\
\hline Study & OR & $95 \% \mathrm{Cl}$ & & & Forest plot \\
\hline $\begin{array}{l}\text { Michelek et } \\
\text { al. } .^{23}\end{array}$ & 0.81 & $(0.7 \mid-I .15)$ & 1.24 & 0.215 & \\
\hline Zaitsu et al. ${ }^{19}$ & 1.04 & $(0.96-I .12)$ & 1.09 & 0.271 & \\
\hline Peters et al. ${ }^{20}$ & 1.63 & $(1.33-1.96)$ & 5.72 & $0.00 * *$ & \\
\hline $\begin{array}{l}\text { Saint-Jacques } \\
\text { et al. }{ }^{22}\end{array}$ & 1.808 & $(1.34-2.43)$ & 3.88 & 0.00 ** & \\
\hline $\begin{array}{l}\text { Michalek et } \\
\text { al. }{ }^{18}\end{array}$ & 1.842 & $(1.53-2.18)$ & 6.64 & $0.00^{* *}$ & \\
\hline $\begin{array}{l}\text { Callahan et } \\
\text { al. }{ }^{24}\end{array}$ & 0.95 & $(0.84-1.37)$ & 0.51 & 0.486 & \\
\hline Pooled $^{31}$ & 1.04 & $(0.94-1.34)$ & 1.78 & 0.108 & \\
\hline
\end{tabular}

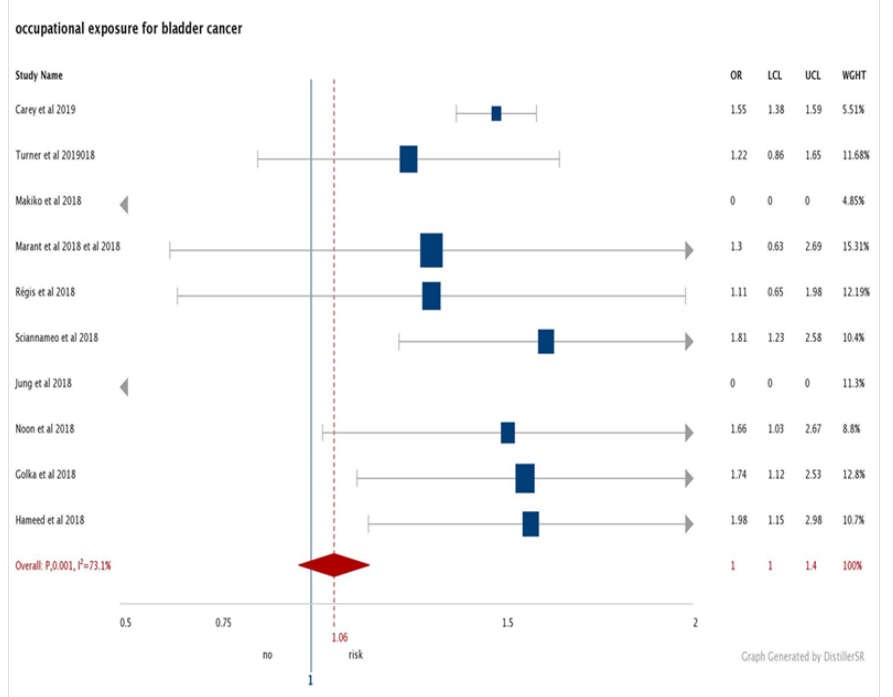

Figure 5Forest plot of association occupational exposure and bladder cancer. Box sizes reflect the weights of studies included in the meta-analysis, horizontal lines are the $95 \% \mathrm{Cls}$, and the summary OR is represented by the diamond. OR: odds ratio, $\mathrm{Cl}$ : confidence interval.

Table 7Distribution of demographic data of bladder cancer studies

\begin{tabular}{|c|c|c|c|c|c|}
\hline Study & $\mathbf{N}$ & AGE & SEX & & Smoking \\
\hline & & & Male & Female & \\
\hline Carey et al. ${ }^{25}$ & III 458 cases & NA & NA & NA & $54.60 \%$ \\
\hline Turner et al. ${ }^{26}$ & 938 cases & NA & NA & NA & NA \\
\hline Makiko et al. ${ }^{27}$ & 10 cases & $56.0 \pm 10.25$ & NA & NA & NA \\
\hline Marant et al. ${ }^{28}$ & 7905 cases & NA & NA & NA & NA \\
\hline Régis et al. ${ }^{30}$ & 84 cases & $57.12 \pm 10.5$ & $85.00 \%$ & $15.00 \%$ & $41.20 \%$ \\
\hline $\begin{array}{l}\text { Sciannameo } \\
\text { et al. } .^{31}\end{array}$ & 893 cases & $52.87 \pm 10.85$ & NA & NA & $45.00 \%$ \\
\hline Jung et al. ${ }^{32}$ & 193 cases & NA & NA & NA & NA \\
\hline Noon et al. ${ }^{33}$ & 17995 cases & NA & NA & NA & NA \\
\hline Golka et al. ${ }^{34}$ & 400 cases & $65.88 \pm 9.21$ & $75.00 \%$ & $25.00 \%$ & NA \\
\hline $\begin{array}{l}\text { Hameed et } \\
\text { al. }{ }^{35}\end{array}$ & 100 cases & $63.74 \pm 8.65$ & $100.00 \%$ & $0.00 \%$ & $60.00 \%$ \\
\hline
\end{tabular}

Table 8Association and risk of occupational exposure for bladder cancer distribution

\begin{tabular}{|c|c|c|c|c|c|}
\hline Study & OR & $95 \% \mathrm{Cl}$ & $\mathbf{Z}$ & $\mathbf{P}$ & Forest plot \\
\hline $\begin{array}{l}\text { Carey et } \\
\text { al. }^{25}\end{array}$ & 1.55 & $\begin{array}{l}(1.38- \\
1.69)\end{array}$ & 22.32 & $0.00 * *$ & I \\
\hline $\begin{array}{l}\text { Turner et } \\
\text { al. }{ }^{26}\end{array}$ & 1.22 & $\begin{array}{l}(0.86- \\
1.65)\end{array}$ & 1.25 & 0.251 & \\
\hline $\begin{array}{l}\text { Makiko et } \\
\text { al. }{ }^{27}\end{array}$ & NA & NA & NA & NA & \\
\hline $\begin{array}{l}\text { Marant et } \\
\text { al. }{ }^{28}\end{array}$ & 1.3 & $\begin{array}{l}(0.63- \\
2.69)\end{array}$ & 0.73 & 0.461 & \\
\hline $\begin{array}{l}\text { Régis et } \\
\text { al. }^{30}\end{array}$ & 1.11 & $\begin{array}{l}(0.65- \\
1.98)\end{array}$ & 0.39 & 0.68 & \\
\hline $\begin{array}{l}\text { Sciannameo } \\
\text { et } \mathrm{al}^{3}{ }^{3} \mid\end{array}$ & 1.81 & $\begin{array}{l}(1.23- \\
2.58)\end{array}$ & 3.22 & $0.00 I^{* *}$ & \\
\hline Jung et al. ${ }^{32}$ & NA & NA & NA & NA & \\
\hline $\begin{array}{l}\text { Noon et } \\
\text { al. }^{33}\end{array}$ & 1.66 & $\begin{array}{l}(1.03- \\
2.67)\end{array}$ & 2.11 & $0.037^{*}$ & \\
\hline $\begin{array}{l}\text { Golka et } \\
\text { al. }^{34}\end{array}$ & 1.74 & $\begin{array}{l}(1.12- \\
2.53)\end{array}$ & 2.51 & $0.012^{*}$ & \\
\hline $\begin{array}{l}\text { Hameed et } \\
\text { al. } .^{35}\end{array}$ & 1.98 & $\begin{array}{l}(1.15- \\
2.98)\end{array}$ & 2.88 & $0.009 *$ & \\
\hline Pooled ${ }^{31}$ & 1.54 & $\begin{array}{l}(1.44- \\
1.75)\end{array}$ & 23.3 & $0.00 * *$ & \\
\hline
\end{tabular}

\section{Discussion}

This study provides a complete systematic review of the literature and quantitative estimates of the associations between occupational exposure and the risk of $\mathrm{KCa}$ and $\mathrm{BCa}$ in population-based observational studies that were published between January 2018 and May 2019. The results of our meta-analysis revealed no significant association between occupational exposure and $\mathrm{KCa}(\mathrm{OR} 1.04,95 \%$ CI:0.94-1.34), whereas there was a positive association between occupational exposure and $\mathrm{BCa}$ (OR 1.54,95\% CI:1.44-1.75).Wielders are predisposed to $\mathrm{KCa}$ because of the association between $\mathrm{KCa}$ and exposure to iron and nickel present in wielding fumes. ${ }^{17}$ Occupational exposures to potential carcinogens can be prevented through quality control, health and safety surveillance in work places. The association between occupational lead exposure and $\mathrm{KCa}$ risk have not been proven to be significant by previous case control studies. ${ }^{24}$ Exposure to a high level of asbestos is associated with $\mathrm{KCa}$ among Canadian industrial workers. ${ }^{20}$ Individuals working in petroleum industries are predisposed to $\mathrm{KCa}$ because there is a possible association between gasoline, diesel exposure and $\mathrm{KCa}^{21}$ The relationship between occupational risk of direct exposure to aromatic amine and cancer was not significant but higher risks were found for chemical, rubber, dyers and printer workers. ${ }^{1} \mathrm{~A}$ significantly elevated risk of $\mathrm{KCa}$ was found among men in higher occupational class most especially in blue-collar industries (OR, 1.61; 95\% CI, 1.34-1.93)which might be linked to job stress among Japanese professionals. ${ }^{19}$

One of the major occupational risk factors for $\mathrm{BCa}$ is the exposure to aromatic amines. ${ }^{37,38}$ Mathieu et al. ${ }^{39}$ found a significant higher risk of $\mathrm{BCa}$ among workers in a rubber production plant according to the results of their systematic review and meta-analysis ((SRR 1.36; $95 \%$ CI: 1.18, 1.57). According toCarey et al. ${ }^{25}$ cigarette smoking is a notable risk factor for $\mathrm{BCa}$ based on the outcome of the Nordic Occupational Cancer study, smoking cessation should be encouraged by employers. Golka et al. ${ }^{34}$ discovered a higher risk of $\mathrm{BCa}$ among former hard coal miners in a former area of coal, iron and steel 
industries in Dortmund, Germany, $70 \%$ of BCa cases were attributed to glutathione S-transferase M1 (GSTM1) negative. From the results of a meta-analysis conducted byFang et al. ${ }^{40}$ based on 506 casecontrol studies GSTM1 was associated with a significantly increased risk in cancer especially in smokers $(\mathrm{OR}=1.17 ; 95 \% \mathrm{CI}=1.14-1.21)$. Indicating that smoking increases the production of oxidative stress, especially in people carrying GSTM1 null genotype.These individuals are more prone to gene damage and hence increases the risk of cancer.

According to Koutroset al. ${ }^{41}$ There was no significant association between occupational exposure to fumigants or fungicides and $\mathrm{BCa}$, with the exception of a positive association among smokers using carbon tetrachloride/carbon disulfide, which was based on only three exposed cases whereas Zhen et al. ${ }^{42}$ conducted quantitative analysis in order to evaluate the relationship between pesticide exposure and the risk of $\mathrm{BCa}$ by summarizing the results of published casecontrol and cohort studies, they observed that pesticide exposure was associated with an increased risk of $\mathrm{BCa}(\mathrm{OR}=.649,95 \%$ CI 1.223 2.223 ), in subgroup analysis, they observed that pesticide exposure is a significant risk factor for $\mathrm{BCa}$ in America $(\mathrm{OR}=1.741,95 \% \mathrm{CI}$ 1.270-2.388), the same result were observed in both case-control group and cohort group (OR=2.075, 95\% CI 1.183-3.638, OR1.146, 95\% CI 1.074-1.223, respectively).

In order to minimize the risk of $\mathrm{KCa}$ and $\mathrm{BCa}$ in working places most especially in hydrocarbon Industries, there is need for industries to design a health, safety and quality control protocols in order to achieve the standard goal of regulating the amount of carcinogenic materials utilized for production purposes and also by modelling the production processes to reduce the release of carcinogens into the environment. ${ }^{43}$ Industries should also endeavor to provide health and safety information for workers as well as setting up smoking cessation interventions at working place. Future studies are required to investigate and address the relationship between occupational exposures and risk of $\mathrm{KCa}$ and $\mathrm{BCa}$, most especially in Hydrocarbon and Metallurgical industries. There is need for more policies and strategies to mitigate the effects of industrial chemicals on health of workers in order to minimize the risk of cancer. Moreover a global epidemiological surveillance system and database should be set up with aim of registering and analyzing reported occupational risk of $\mathrm{KCa}$ and $\mathrm{BCa}$.

\section{Limitation}

There are some limitations in the present study. The gender effects of exposure to potential occupational carcinogens could not be analyzed in the present study, because majority of the selected studies did not report on gender effects. Moreover, there was no toxicological analyses in most of the studies.

\section{Conclusion}

From the result of our qualitative and quantitative analysis there was no significant association between occupational exposure and $\mathrm{KCa}$, whereas there was a significant association between occupational exposure and $\mathrm{BCa}$.

\section{Acknowledgments}

None.

\section{Conflicts of interest}

The author declares there is no conflict of interest.

\section{References}

1. Claire MM, Kevin DS, Isabelle B, et al. Occupational exposures and cancer: a review of agents and relative risk estimates. Occup Environ Med. 2018;75(8):604-614.

2. Saeed Y, Ayda FA, Mohammad N. Occupational and Environmental Cancer. Asian Pac J Environment and Cancer. 2018;1(1):5-13.

3. Mark PP, Patricia AS, Melissa CF, et al. Occupational exposure to chlorinated solvents and kidney cancer: a case-control study. Occup Environ Med. 2017;74(4):268-274.

4. Elin M, Johann PI, Eirikur J, et al. Occupation as a risk factor for renal cell cancer: a nationwide, prospective epidemiological study. Scand J Urol Nephrol. 2016;50(3):181-185.

5. Xie B, Hu Y, Liang Z, et al. Association between pesticide exposure and risk of kidney cancer: a meta-analysis. Onco Targets Ther. 2016;9:38933900 .

6. MacLeod JS, Harris MA, Tjepkema M, et al. Cancer Risks among Welders and Occasional Welders in a National Population-Based Cohort Study: Canadian Census Health and Environmental Cohort. Saf Health Work. 2017;8(3):258-266.

7. Mahdavifar N, Mohammadian M, Ghoncheh M, et al. Incidence, mortality and risk factors of kidney cancer in the world. WCRJ. 2018;5:(1)9:1-9.

8. Kishor H, Jan IM, Elisabete W, et al. Occupational exposure to solvents and bladder cancer: A population-based case control study in Nordic countries. Int $j$ cancer. 2017;140(8):1736-1746.

9. Umberto C, Karim B, Axel B, et al. Epidemiology of renal cell carcinoma. Eur Urol. 2019;75:74-84.

10. Yu MH, W JC, Ying CL, et al. Adiponectin gene polymorphisms and obesity increase the susceptibility to arsenic-related renal cell carcinoma. Toxicol Appl Pharmacol. 2018;350:11-20.

11. Zaitsu M, Kaneko R, Takeuchi T, et al. Occupational class and male cancer incidence: Nationwide, multicenter, hospital-based case-control study in Japan. Cancer Med. 2019;8(2):795-813.

12. Alexa RM, Mohamad EA, Michael AG. Epidemiology and Risk Factors of Renal Cell Carcinoma. Diagnosis and Surgical Management of Renal Tumors. 2019;1-11.

13. Anas MS, Mohamed MG, Muneer JH, et al. Trends in renal-cell carcinoma incidence and mortality in the United States in the last 2 decades: a SEERbased study. Clin Genitourin Cancer. 2019;17(1):46-57.

14. Marcus GC, Ibrahim J, Peter CB, et al. Epidemiology of bladder cancer: a systematic review and contemporary update of risk factors in 2018. Eur Urol. 2018;74(6):784-795.

15. Kishor H, Jan IM, Elisabete W, et al. P032 Occupational solvent exposure and risk of bladder cancer in the Nordic countries. Occup Environ Med. 2016;73(1):A131-A131.

16. Moher D, Liberati A, Tetzlaff J, et al. Preferred Reporting Items for Systematic Reviews and Meta-Analyses: The PRISMA Statement. Ann Intern Med. 2009;151(4):264-269.

17. Michalek IM, Martinsen JI, Weiderpass E, et al. Heavy metals, welding fumes, and other occupational exposures, and the risk of kidney cancer: A population-based nested case-control study in three Nordic countries. Environ Res. 2019;173:117-123.

18. Michalek IM, Martinsen JI, Weiderpass E, et al. Occupation and Risk of Kidney Cancer in Nordic Countries. J Occup Environ Med. 2019;61(1):4146.

19. Zaitsu M, Cuevas AG, Trudel FC, et al. Occupational class and risk of renal cell cancer. Health Sci Rep. 2018;1(6):e49. 
20. Peters CE, Parent MÉ, Harris SA, et al. Workplace exposure to asbestos and the risk of kidney cancer in Canadian men. Can J Public Health. 2018;109(4):464-472.

21. Peters CE, Parent MÉ, Harris SA, et al. Occupational Exposure to Diese and Gasoline Engine Exhausts and the Risk of Kidney Cancer in Canadian Men. Ann Work Expo Health. 2018;62(8):978-989.

22. Saint-Jacques N, Brown P Nauta L, Boxall J, et al. Estimating the risk of bladder and kidney cancer from exposure to low-levels of arsenic in drinking water, Nova Scotia, Canada. Environ Int. 2018;110:95-104.

23. Michalek IM, Martinsen, JI, Weiderpass E, et al. Occupation and risk of cancer of the renal pelvis in Nordic countries. BJU Int. 2019;123(2):233238.

24. Callahan CL, Friesen MC, Locke SJ, et al. Case-control investigation of occupational lead exposure and kidney cancer. Occup Environ Med. 2019;76(7):433-440.

25. Carey RN, Fritschi L, Driscoll TR, et al. Interventions to reduce future cancer incidence from diesel engine exhaust: What might work? Cancer Prev Res. 2019;12(1):13-20.

26. Turner MC, Gracia-Lavedan E, Cirac M, et al. Ambient air pollution and incident bladder cancer risk: Updated analysis of the Spanish Bladder Cancer Study. Int J Cancer. 2019;145(4):894-900.

27. Makiko N, Kazuyuki O, Yoko E, Satoko I, Kota F, Noriyuki Y, et al. Epidemic of bladder cancer in Japanese male workers exposed mainly to ortho-toluidine. Occup Environ Med. 2019;76(1):A93-A93.

28. Marant MC, Shield KD, Vignat J, et al. Cancers in France in 2015 attributable to occupational exposures. Int $J$ Hyg Environ Health. 2019;222(1):22-29.

29. Carta A, Pavanello S, Mastrangelo G, et al. Impact of Occupational Exposures and Genetic Polymorphisms on Recurrence and Progression of Non-Muscle-Invasive Bladder Cancer. Int J Environ Res Public Health. 2018;15(8):1563.

30. Régis C, Michel G, Pascal W, et al. Bladder cancer and occupational exposure to metalworking fluid mist: a counter-matched case-control study in French steel-producing factories. Occup Environ Med. 2018;75(5):328336.

31. Sciannameo V, Carta A, d'Errico A, et al. New insights on occupational exposure and bladder cancer risk: a pooled analysis of two Italian casecontrol studies. Int Arch Occup Environ Health. 2019;92(3):347-359.
32. Jung YL, Tompa E, Longo C, et al. The Economic Burden of Bladder Cancer Due to Occupational Exposure. J Occup Environ Med. 2018;60(3):217-225.

33. Noon AP, Martinsen JI, Catto JWF, et al. Occupation and Bladder Cancer Phenotype: Identification of Workplace Patterns That Increase the Risk of Advanced Disease Beyond Overall Incidence. Eur Urol Focus. 2018;4(5):725-730.

34. Golka K, Ovsiannikov D, Krech E, et al. 364 Urinary bladder cancer in a former area of coal, iron and steel industries in Germany. Occup Environ Med. 2018;75(2):A402.2-A402.

35. Hameed DA, Yassa HA, Agban MN, et al. Genetic aberrations of the $\mathrm{K}$-ras proto-oncogene in bladder cancer in relation to pesticide exposure. Environ Sci Pollut R. 2018;25(22):21535-21542.

36. Von Elm E, Altman D, Egger M, et al. The Strengthening the Reporting of Observational Studies in Epidemiology (STROBE) statement: Guidelines for reporting observational studies. Ann Intern Med. 2007;147:573-577.

37. Case RAM, Hosker ME. Tumours of the urinary bladder as an occupational disease in the rubber industry in England and Wales. Br J Prev Soc Med. 1954;8:39-50

38. Chen $\mathrm{HI}$, Liou SH, Loh CH, et al. Bladder cancer screening and monitoring of 4, 4'-methylenebis (2-chloroaniline) exposure among workers in Taiwan. Urology. 2005;66:305-310.

39. Mathieu B, Alice K, Peter B. Meta-analysis of occupational exposures in the rubber manufacturing industry and risk of cancer. Int J Epidemiol. 2017;46(6):1940-1947.

40. Fang J, Wang S, Zhang S, et al. Association of the glutathione s-transferase $\mathrm{m} 1, \mathrm{t} 1$ polymorphisms with cancer: evidence from a meta-analysis. PLoS One. 2013;8(11):e78707

41. Koutros S, Silverman DT, Alavanja MC, et al. Occupational exposure to pesticides and bladder cancer risk. Int J Epidemiol. 2016;45(3):792-805.

42. Liang Z, Wang X, Xie B, et al. Pesticide exposure and risk of bladder cancer: A meta-analysis. Oncotarget. 2016;7(41):66959-66969.

43. John WC. Reducing occupational exposure to chemical carcinogens. Occup Med. 2009;59(2):96-100. 